\title{
Intrapulmonary mature teratoma: case report of an exceptional entity
}

\begin{abstract}
Teratomas are tumors consisting of tissues derived from more than one germ cell line. Criteria for pulmonary origin are exclusion of a gonadal or other extra-gonadal primary site and origin entirely within the lung. The intrapulmonary teratoma (IPT) is a rare type of teratoma extra-gonadal which often presents with nonspecific symptoms and wrongly can be diagnosed as other diseases. Here we report a patient with IPT who was initially misdiagnosed as pulmonary aspergilloma.
\end{abstract}

We report a case of mature pulmonary teratoma in a 22-year-old male with no toxic habits, treated in 2007 for smear-positive pulmonary tuberculosis, presenting progressively for 1 year, with a left chest pain, intermittent episodes of cough and hemoptysis, and dyspnea. A computed tomography (CT) chest scan showed a heterogeneous tumor with cavitary lesion in the left upper lobe. The patient had a left upper lobectomy with complete resection of the mediastinal mass. The patient is currently in good general condition, and does not present any complications or recurrence and thyroid function was normal during the long term follow up.

Preoperative diagnosis of IPT is not always possible and is usually misdiagnosed early on due to its rarity, nonspecific and vague symptoms, normal lab results, and indistinguishable chest $\mathrm{x}$-ray results. We report a case of intrapulmonary teratoma in a 22-year-old man and review the relevant literature.

Keywords: mature, teratoma, lung, surgery, rare case
Volume 8 Issue | - 202|

\author{
Najat Id el haj, ${ }^{1,2}$ S. Boubia, ${ }^{1,2}$ M. Ridai ${ }^{1,2}$ \\ 'Department of thoracic surgery, University Hospital Center \\ Ibn Rochd Casablanca, Morocco \\ ${ }^{2}$ Hassan II University of Casablanca, Morocco
}

\begin{abstract}
Correspondence: Najat Id el haj, Department of thoracic surgery, University Hospital Center Ibn Rochd Casablanca, Morocco,Tel 2126627|3360,Email najatidelhaj@yahoo.fr
\end{abstract}

Received: January 07, 2021 | Published: Febrauary 05, 2021

\begin{abstract}
Abbreviations: Intrapulmonary Teratoma

CT, Computed Tomography;

IPT,

\section{Introduction}

Intrapulmonary teratoma originates from totipotential cells of one or more of the three germinative layers. They may be solid or cystic, mature or immature. Intrathoracic teratomas are usually seen in the mediastinum. However, in some extremely rare cases, intrathoracic teratomas occur in the parenchyma of the lungs. Since its first description by Mohr in 1839 in the literature, only 65 cases of intrapulmonary teratoma have been collected, until $1996 .{ }^{1}$
\end{abstract}

\section{Case report I}

A 22-years-old nonsmoker male treated of pulmonary tuberculosis in 2007 presented with a one-year history of intermittent episodes of cough and hemoptysis, thoracic left pain, dyspnea induced by high activity, weight loss, fever, and night sweats. A computed tomography (CT) chest scan showed a heterogeneous tumor with cavitary lesion, occupying the left upper lobe. It appeared to be adherent to the aortic arch and the level of the main pulmonary trunk (figure 1). Bronchoscopy revealed a very important inflammation of the left bronchial tree (3rd degree), with bleeding at least contact, and the muco-purulent secretions whose bacteriological and mycotic examination is unremarkable. Bronchial biopsy revealed a nonspecific chronic inflammation. Pulmonary function test results showed mild obstructive ventilatory disorder limited to small aerial ducts; with a forced expiratory volume in one second of $82 \%$. The aspergillum serology was negative. Routine laboratory testing was entirely normal including testing for koch bacillus in sputum. The diagnosis of pulmonary aspergilloma in its complex form was suspected, the patient initially prepared with an antibiotic and hemostatic treatment before the surgery. A left posterior-lateral thoracotomy was realized, the exploration discovers a bulky hard yellowish tumoral mass of approximately $7 \mathrm{~cm}$, was identified in the culmen. There was connection to mediastinal fat, and lingula was completely hepatized. the lung was completely adherent to see a total symphysis especially at the level of the apex making the adhesiolysis and the dissection of the hilum very difficult and bleeding. We opted for a left upper lobectomy carrying in the monobloc mediastinal mass. The analysis of the operative piece shows a cystic tumoral process with thick wall, pilous and friable contents. The patient presented favorable evolution and was discharged on postoperative day 5. The final histological study concluded that it was a primary intrapulmonary mature teratoma with complete resection of the tumor. Follow up during the next years showed no sign of recurrence.

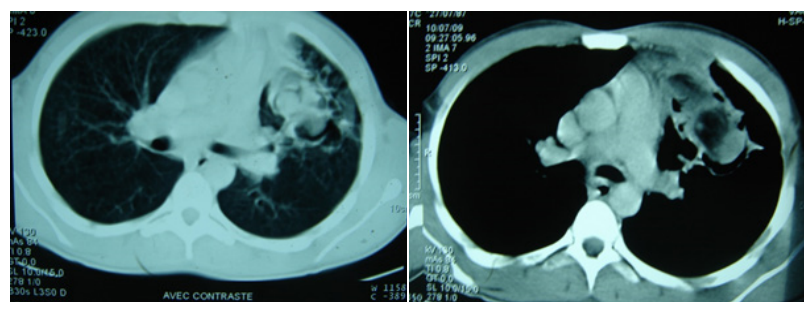

Figure I Heterogeneous tumor with cavitary lesion, occupying the left upper lobe; adherent to the aortic arch and the level of the main pulmonary trunk.

\section{Discussion}

Teratomas are tumors originating from totipotential cells, 
the mature teratoma include only well differentiated tissues. Intrapulmonary teratomas are extremely rare, it is necessary to exclude the possibility of a mediastinal teratoma or metastasis from an extrapulmonary germ cell tumor. ${ }^{2,3}$ Most intrapulmonary teratomas have occurred in first or second decade of life, with ages ranging from 10 to 68 years. The prevalence of intrapulmonary teratoma in women and men is similar., ${ }^{4,5}$ Teratoma frequently occur in the upper left lobe, mainly the ventral segment of the upper lobe (approximately 65\%). ${ }^{1,3}$ As it was to observe in our case. A preoperative clinical diagnosis is difficult because of some nonspecific symptoms, such as chest pain, hemoptysis, cough, loss weight, fever, and features of pneumonia or bronchiectasis. The bronchoscopy evidence of hair in the bronchial tree or the clinical equivalent of trichophytisis is considered a suggestive symptom. However, trichophytisis has been reported in seven patients only. ${ }^{4}$ Radiographically, lesions are typically cystic masses often with focal calcification. ${ }^{6,7}$ Computed tomography accurately estimates the density of all elements such as soft tissue, fluid, fat, calcification and teeth, and is extremely valuable for the differentiation between a ruptured and an unruptured teratoma. ${ }^{8}$ It is the case of our observations which the radiological diagnosis was difficult. Surgical resection is the treatment of choice; and radical extirpation leads to a long recurrence-free survival, because of its malignant potential, possibility for rupture; and high percentage approximately $30 \%$ of malignant teratomas; options may range from segmentectomy to pneumonectomy. ${ }^{2,4}$ The anatomopathological study is the definitive diagnostic method. Pulmonary teratomas are mostly composed of mature, cystic somatic tissue, although malignant elements may occur. Mature elements often take the form of squamous lined cysts. Thymic or pancreatic elements may be seen in mature teratoma. ${ }^{8}$ Tumor rupture, hemoptysis, compression of the airways and malignant transformation are complications of IPT if it is not managed surgically. ${ }^{9}$ About $80 \%$ of teratoma contain only tissue components and are considered benign. In the adult population, the presence of immature tissue components is associated with a poor prognosis. In rare cases, teratoma can undergo malignant transformation. ${ }^{10}$

\section{Conclusion}

Intrapulmonary mature teratoma is an exceptionally rare tumor. The diagnosis has to rely on the clinic and radiologic imaging. Definitive confirmation of the diagnosis is made through anatomopathological examination. Surgery with complete resection is considered the standard curative treatment to prevent complications and malignant transformation and to have good long-term prognosis.

\section{Acknowledgement}

None

\section{Conflicts of interest}

All authors declare that there is no conflicts of interest.

\section{References}

1. Asano S, Hoshikawa Y, Yamane Y, et al. An intrapulmonary teratoma associated with bronchectasia containing various kinds of primordium: a case report and review of the literature. Virchows Arch. 2000;436(4):384 -384 .

2. Sandeep SR, Naveen S, Sudhir M, et al. Intrapulmonary teratoma: An exceptional disease. Ann Thoracic Surg. 2007; 83(3):1194-1196.

3. Morgan DE, Sanders C, Mcelvein RB, et al. Intrapulmonary teratoma: a case report and review of the literature. J Thorac Imag. 1992;7(3):70-77.

4. Zenker D, Aleksic I. Intrapulmonary cystic benign teratoma: A case report and review of the literature. Ann Thoracic Cardiovasc Surg. 2004;10(5):290-292.

5. Carter D, Eggleston JC. Hartmann WH. ed. Tumor of the lower respiratory tract. Washington, Ds: Armed forces institute of pathology, 1980, pp. 325326.

6. Trivedi SA, Mehta KN, Nanavaty JM. Teratoma of the lung: report of a case. Br J Dis Chest. 1966:60:156-159.

7. Bateson EM, Hayes JA, Woo MM. Endobronchial teratoma associated with bronchectasis. Thorax.1968;23(1):69-76.

8. Ricardo Alexandre faria, José Alexandre Bizon, Roberto Saad Junior, et al. Intrapulmonary teratoma: case report. J Bras Pneumologie. 2007;33(5):612-615.

9. Mardani P, Naseri N, Amirian A, et al. Intrapulmonary mature cystic teratoma of the lung: case report of a rare entity. BMC Surgery. 2020;20(1):203.

10. Ditah $\mathrm{C}$, Templin T, Mandal R, et al. Isolated intrapulmonary teratoma. $J$ Thorac Cardiovasc Surg. 2016;152(6):e129-e131. 